\title{
Botryosphaeriaceae from palms in Thailand - Barriopsis archontophoenicis sp. nov, from Archontophoenix alexandrae
}

\section{Konta $\mathrm{S}^{1,3}$, Phillips AJL ${ }^{4}$, Bahkali $\mathrm{AH}^{5}$, Jones EBG ${ }^{5}$, Eungwanichayapant $\mathrm{DP}^{3}$, Hyde $\mathrm{KD}^{1,2,3,5}$ and Boonmee $S^{1}$}

${ }^{1}$ Center of Excellence in Fungal Research, Mae Fah Luang University, Chiang Rai 57100, Thailand

${ }^{2}$ Key Laboratory for Plant Biodiversity and Biogeography of East Asia, Kunming Institute of Botany, Chinese Academy of Science, Kunming 650201, Yunnan, China

${ }^{3}$ School of Science, Mae Fah Luang University, Chiang Rai. 57100, Thailand

${ }^{4}$ University of Lisbon, Faculty of Sciences, Biosystems and Integrative Sciences Institute (BioISI), Campo Grande, 1749-016 Lisbon, Portugal

${ }^{5}$ Department of Botany and Microbiology, College of Science, King Saud University, P.O. Box: 2455, Riyadh 1145, Saudi Arabia

Konta S, Phillips AJL, Bahkali AH, Jones EBG, Eungwanichayapant PD, Hyde KD, Boonmee S 2016 - Botryosphaeriaceae from palms in Thailand - Barriopsis archontophoenicis sp. nov, from Archontophoenix alexandrae. Mycosphere 7(7), 921-932, Doi 10.5943/mycosphere/si/1b/1

\begin{abstract}
During our studies of palm fungi in Thailand we identified a new species of Barriopsis on a petiole of Archontophoenix alexandrae, which we introduce herein as B. archontophoenicis. The new species is compared with other species in the genus Barriopsis and differs in its epapillate ostiole and smaller ascospores. Phylogenetic analyses of combined ITS, LSU, SSU and TEF1- $\alpha$ sequence data also show the species to be distinct.
\end{abstract}

Key words - ascospores - molecular phylogeny - morphology - palm fungi - taxonomy

\section{Introduction}

Hyde and co-authors have been studying the fungi on palms since 1988 (Hyde 1988, Hyde et al. 2000, Fröhlich and Hyde 2000, Taylor \& Hyde 2003) and this paper is a continuation of that research. In earlier studies, all taxa were described and arranged in the Ascomycota based on morphology. This approach was, however, subjective and many taxa were wrongly placed or placed in Ascomycota genera incertae sedis. It is therefore essential that these palm fungi are recollected, epitypified where needed, isolated and sequence data obtained so that the palm fungi can be placed in a natural taxonomic framework (Ariyawansa et al. 2014a, b, Jayasiri et al. 2015). In this paper, we introduce a new Barriopsis species collected on Archontophoenix in Thailand. It is characterized on the basis of morphology and DNA sequence data. The new species is illustrated and compared with other species in the genus. 


\section{Materials \& Methods}

\section{Collection, isolation and identification}

Fresh material of Archontophoenix alexandrae was collected from Chiang Mai, Thailand in 2014. Fungal structures were examined with a Motic SMZ 168 series stereomicroscope and photographed with an Axio camera on a Zeiss Discover V8 stereomicroscope. Micromorphological structures were photographed with a Canon 600D camera on a Nikon ECLIPSE 80i microscope. Fungal structures were measured with Image Frame Work (IFW) version 0.9.7. Photoplates were made with Adobe Photoshop CS3 Extended version 10. Isolations were made from single ascospores following the method of Chomnunti et al. (2014). Growth rates were recorded by measuring colony diam. after 7 days of incubation at $25^{\circ} \mathrm{C}$ on MEA medium. The holotype specimen is deposited in Herb. MFLU and ex-type culture in MFLUCC at Mae Fah Luang University, Chiang Rai, Thailand. Facesoffungi and Index Fungorum numbers are registered as outlined in Jayasiri et al. (2015) and Index Fungorum (2016).

\section{Fungal DNA extraction and $P C R$ reaction}

Genomic DNA was extracted from fresh mycelium grown on MEA for two weeks using the Biospin Fungus Genomic DNA Extraction Kit (BioFlux $®)$ (Phukhamsakda et al. 2015). Specific rDNA regions were amplified with different gene primers, i.e. LROR and LR5 to amplify the large subunit rDNA (LSU) (Vilgalys \& Hester 1990), NS1 and NS4 to amplify region of nuclear small subunit rDNA (SSU), ITS5 and ITS4 to amplify the internal transcribed spacers (ITS) (White et al. 1990), and a fragment of translation elongation factor 1- $\alpha$ (TEF1- $\alpha$ ) was amplified and sequenced using EF1-526F and EF1-1567R (Jacobs et al. 2004). The amplification was performed with an initial denaturing step of $5 \mathrm{~min}$ at $95^{\circ} \mathrm{C}$, followed by 35 cycles of $30 \mathrm{~s}$ at $94^{\circ} \mathrm{C}, 30 \mathrm{~s}$ annealing at $52^{\circ} \mathrm{C}, 56^{\circ} \mathrm{C}$ for ITS and TEF1- $\alpha$ respectively, then $1 \mathrm{~min}$ at $72^{\circ} \mathrm{C}$, and a final extension of $7 \mathrm{~min}$ at $72^{\circ} \mathrm{C}$ (Doilom et al. 2014). PCR products were checked on $1 \%$ agarose electrophoresis gels stained with ethidium bromide. PCR products were sequenced by Shangkai Majorbio Biopharm Technology Co, Ltd, China.

\section{Sequence alignment and phylogenetic analyses}

DNA sequences were checked with BioEdit (Hall 1999) and MEGA6 (Tamura et al. 2013). A BLAST search with the LSU sequence data was used to reveal the closest matching taxa in Botryosphaeriaceae (Liu et al. 2014, Phookamsak et al. 2015). Multiple sequence alignments were performed with MAFFT (Katoh et al. 2013) alignment online (http://www.ebi.ac.uk/Tools/msa/mafft/). ITS, LSU, SSU and TEF sequences datasets were first analyzed separately and then the individual datasets were concatenated into a combined dataset and prepared in Mega v.6 (Tamura et al. 2013). Data were converted from fasta to nexus format with Clustal X (Thompson et al. 1997). Maximum-parsimony (MP) analysis was done with PAUP v. $4.0 \mathrm{~b} 10$ (Swofford 2002) and robustness of the branches was determined with 1000 bootstrap replicates along with 1000 of max-trees. Maximum likelihood analysis was performed by RAxMl GUIv.0.9b2 (Kishino \& Hasegawa 1989, Silvestro \& Michalak 2010). The search strategy was set to rapid bootstrapping at 1000 and the analysis carried out using the GTR-GAMMA model of nucleotide substitution. The number of replicates was inferred using the stopping criterion. Bootstrap values greater than $50 \%$ were accepted. The model of evolution was determined with MrModeltest 2.2 (Nylander 2004) and posterior probabilities (PP) (Rannala \& Yang 1996, Zhaxybayeva \& Gogarten 2002) were determined by Markov Chain Monte Carlo sampling (BMCMC) using MrBayes v3.1.2 (Huelsenbeck \& Ronquist 2001). Bayesian posterior probabilities (BYPP) greater than $95 \%$ were accepted. The phylogenetic tree was visualized with Tree View32 (Page 1996). 
Table 1 GenBank accession numbers of the sequences used in phylogenetic analysis.

\begin{tabular}{|c|c|c|c|c|c|}
\hline \multirow{2}{*}{ Species name } & \multirow{2}{*}{ strain } & \multicolumn{4}{|c|}{ GenBank accession number } \\
\hline & & ITS & LSU & SSU & TEF \\
\hline Alanphillipsia aloeicola & CBS:138896 & KP004444 & KP004472 & - & - \\
\hline Alanphillipsia aloeigena & CPC 21286 & KF777137 & KF777193 & - & - \\
\hline Alanphillipsia aloes & CPC 21298 & KF777138 & KF777194 & - & - \\
\hline Alanphillipsia aloetica & CPC 21109 & KF777139 & KF777195 & - & - \\
\hline Alanphillipsia euphorbiae & CPC 21628 & KF777140 & KF777196 & - & - \\
\hline $\begin{array}{l}\text { Barriopsis } \\
\text { archontophoenicis }\end{array}$ & MFLUCC 14-1164 & KX235306 & KX235307 & KX235308 & KX235305 \\
\hline Barriopsis fusca & CBS 174.26 & EU673330 & NG_042419 & EU673182 & EU673296 \\
\hline Barriopsis iraniana & IRAN1448C & KF766150 & KF7̄66318 & KF766231 & FJ919652 \\
\hline Barriopsis iraniana & IRAN1451C & FJ919668 & - & - & FJ919657 \\
\hline Barriopsis tectonae & MFLUCC 12-0381 & KJ556515 & - & - & KJ556516 \\
\hline Diplodia mutila & CBS 112553 & AY259093 & - & EU673213 & AY573219 \\
\hline Diplodia mutila & CBS 230.30 & DQ458886 & EU673265 & EU673214 & DQ458869 \\
\hline Diplodia seriata & CBS 112555 & AY259094 & - & KF766244 & AY573220 \\
\hline Diplodia seriata & CBS 119049 & DQ458889 & EU673266 & EU673216 & DQ458874 \\
\hline Diplodia spegazziniana & CBS 302.75 & EU673319 & EU673238 & EU673168 & EU673286 \\
\hline Dothiorella moneti & MUCC505 & EF591920 & EF591937 & - & EF591971 \\
\hline Dothiorella moneti & MUCC507 & EF591922 & EF591939 & - & EF591973 \\
\hline Dothiorella sarmentorum & IMI $63581 b$ & AY573212 & - & - & AY573235 \\
\hline Dothiorella sarmentorum & CBS 115038 & AY573206 & DQ377860 & KF766248 & AY573223 \\
\hline Lasiodiplodia gonubiensis & CMW14077 & AY639595 & - & - & DQ103566 \\
\hline Lasiodiplodia gonubiensis & $\begin{array}{l}\text { CMW14078 } \\
\text { /CBS115812 }\end{array}$ & AY639594 & - & - & DQ103567 \\
\hline Lasiodiplodia margaritacea & $\begin{array}{l}\text { CBS122519 } \\
116355\end{array}$ & EU144050 & - & - & EU144065 \\
\hline Lasiodiplodia margaritacea & CBS 122519 & EU144050 & - & - & EU144065 \\
\hline Lasiodiplodia margaritacea & CBS 122065 & EU144051 & - & - & EU144066 \\
\hline $\begin{array}{l}\text { Lasiodiplodia } \\
\text { pseudotheobromae }\end{array}$ & CBS 116459 & EF622077 & EU673256 & KF766279 & EF622057 \\
\hline $\begin{array}{l}\text { Lasiodiplodia } \\
\text { pseudotheobromae }\end{array}$ & CBS 447.62 & EF622081 & EU673255 & EU673198 & EF622060 \\
\hline Lasiodiplodia theobromae & CBS 164.96 & AY640255 & NG 042460 & EU673196 & AY640258 \\
\hline Lasiodiplodia theobromae & CBS 124.13 & DQ458890 & AY928054 & EU673195 & DQ458875 \\
\hline Neodeightonia palmicola & MFLUCC 10-0822 & HQ199222 & HQ199221 & HQ199223 & - \\
\hline Neodeightonia palmicola & MFLUCC10-0823 & HQ199224 & HQ199225 & HQ199226 & - \\
\hline Neodeightonia phoenicum & CBS 122528 & KF766198 & EU673261 & KF766285 & EU673309 \\
\hline Neodeightonia phoenicum & CBS 123168 & EU673339 & EU673260 & EU673204 & EU673308 \\
\hline Neodeightonia phoenicum & CBS 169.34 & EU673338 & EU673259 & EU673203 & EU673307 \\
\hline Neodeightonia subglobosa & CBS 448.91 & EU673337 & DQ377866 & KF766286 & EU673306 \\
\hline Phaeobotryon cupressi & IRAN1455C & FJ919672 & - & - & FJ919661 \\
\hline Phaeobotryon cupressi & IRAN1456C & FJ919670 & - & - & FJ919659 \\
\hline Phaeobotryon mamane & CPC 12440 & EU673332 & NG 042459 & EU673184 & EU673298 \\
\hline Phaeobotryon mamane & CPC 12442 & EU673333 & DQ377899 & & EU673299 \\
\hline $\begin{array}{l}\text { Pseudofusicoccum } \\
\text { stromaticum }\end{array}$ & CBS 117448 & KF766223 & DQ377931 & EU673146 & - \\
\hline $\begin{array}{l}\text { Pseudofusicoccum } \\
\text { stromaticum }\end{array}$ & CBS 117449 & - & DQ377932 & EU673147 & \\
\hline Spencermartinsia viticola & CBS 117009 & AY905554 & NG 042420 & KF766313 & AY905559 \\
\hline Sphaeropsis citrigena & ICMP 16812 & EU673328 & NG 042458 & EU673180 & EU673294 \\
\hline Sphaeropsis citrigena & ICMP 16818 & EU673329 & EU673247 & EU673181 & EU673295 \\
\hline Sphaeropsis eucalypticola & MFLUCC 11-0579 & JX646802 & NG 042727 & JX646835 & JX646867 \\
\hline Sphaeropsis eucalypticola & MFLUCC 11-0654 & JX646803 & JX646820 & JX646836 & JX646868 \\
\hline Sphaeropsis porosa & $\begin{array}{l}\text { CBS 110496/ STE-U } \\
5132\end{array}$ & KF766210 & DQ377894 & EU673179 & AY343340 \\
\hline Sphaeropsis visci & CBS 186.97 & KF766211 & EU754216 & EU754117 & EU673293 \\
\hline Sphaeropsis visci & CBS 100163 & EU673324 & EU754215 & EU754116 & EU673292 \\
\hline
\end{tabular}




\begin{abstract}
Abbreviation: CBS: Centraalbureau voor Schimmelcultures, The Netherlands; CPC: Collection of Pedro Crous housed at CBS; IRAN: Iranian Fungal Culture Collection, Iranian Research Institute of Plant Protection, Iran; MFLUCC: Mae Fah Luang University Culture Collection, Chiang Rai, Thailand; MUCC: Culture Collection, Laboratory of Plant Pathology, Mie University, Tsu, Mie Prefecture, Japan; IMI: CABI Bioscience, Egham, UK; CMW: M.J. Wingfield, FABI, University of Pretoria, South Africa; ICMP: International Collection of Micro-organisms from Plants, Landcare Research, New Zealand; STE-U: Culture collection of the Department of Plant Pathology, University of Stellenbosch, South Africa.
\end{abstract}

\title{
Results
}

\section{Phylogeny}

The combined dataset of 47 taxa with Pseudofusicoccum stromaticum (Botryosphaeriaceae) as the outgroup taxon contained 3505 characters including gaps. Of these, 2729 were constant, 181 were variable and parsimony-uninformative and 595 were parsimony informative. Phylogenetic trees were generated by maximum parsimony (MP), maximum likelihood (ML) and Bayesian posterior probabilities (BYPP). The equally weighted maximum parsimony tree resulted in a single tree of 1415 steps, a consistency index (CI) of 0.728 , a retention index (RI) of 0.848 , a rescaled consistency index (RC) of 0.618 and homoplasy index (HI) of 0.272. Phylogenetic analysis indicated eight major clades in Botryosphaeriaceae (Fig. 1). The new species Barriopsis archontophoenicis clustered with all other Barriopsis species in a well-supported clade.

Barriopsis archontophoenicis Konta, Boonmee \& K.D. Hyde, sp. nov.

Figs 2-4 Index Fungorum number: IF552104

Facesoffungi number: 02097

Etymology - The specific epithet refers to the host genus Archontophoenix.

Holotype - MFLU: 15-0015

Saprobic on woody tissue of Archontophoenix alexandrae. Sexual morph on fresh specimen: Ascostromata 103-194 $\mu \mathrm{m}$, high $\times$ 99-124 $\mu \mathrm{m}$ diam. $(\overline{\mathrm{x}}=132 \times 120 \mu \mathrm{m}, \mathrm{n}=10)$, solitary to aggregated, immersed, erumpent at maturity, uniloculate, subglobose, minutely papillate, some with a short neck, and slightly flattened at the base. Ostiole epapillate. Peridium 5-14 $\mu m$ wide, composed of outer layers of black, thick-walled, cells of textura angularis, inner layers hyaline and thin-walled. Hamathecium comprising numerous, 2-3 $\mu \mathrm{m}$ wide, hypha-like, septate, cellular pseudoparaphyses. Asci 70-144 × 17-25 $\mu m(\overline{\mathrm{x}}=109 \times 20 \mu m, \mathrm{n}=10)$, 8-spored, bitunicate, cylindrical-clavate, short-pedicellate, apically rounded, with an ocular chamber. Ascospores 17-24 $\times 7-10 \mu m(\overline{\mathrm{x}}=22 \times 9 \mu m, \mathrm{n}=20)$, bi-seriate, ellipsoid to ovoid, broad at the middle, aseptate, initially hyaline, becoming dark brown at maturity, with a large central guttule, without terminal apiculi, smooth-walled, with a distinct mucilaginous sheath. Sexual morph formed in culture after 6 months of incubation: Ascostromata 206-478 $\mu \mathrm{m}$ high $\times 322-435 \mu \mathrm{m}$ diam. $(\overline{\mathrm{x}}=321 \times 336 \mu \mathrm{m}, \mathrm{n}=10)$, superficial, covered by mycelium, solitary or gregarious, scattered, uniloculate, globose to subglobose, dark brown to black. Ostiole and Hamathecium not apparent. Peridium comprising several layers of textura angularis, outer layers dark-brown and thick-walled, inner layers hyaline and thin-walled. Ascospores 20-36 $\times 10-16 \mu \mathrm{m}(\overline{\mathrm{x}}=32 \times 14 \mu \mathrm{m}$, $\mathrm{n}=20$ ), ellipsoid to ovoid, initially reddish-brown, becoming dark brown at maturity, 2-septate, with two large guttules in center, thick-walled, smooth-walled, ellipsoid to obovoid, with terminal apiculi at both ends. Asexual morph in culture: Coelomycetous. Conidiomata stromatic, pycnidial, superficial, dark brown to black, covered with dense mycelium, on PDA mostly uniloculate, individual or aggregated, thick-walled, ostiolate. Ostiole central, circular, epapillate. Paraphyses arising from the conidiogenous layer, extending above the level of developing conidia, thin-walled, hyaline, usually aseptate, and tip rounded. Conidiophores absent. Conidiogenous cells

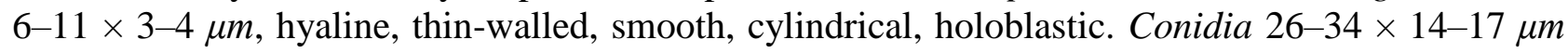
$(\overline{\mathrm{x}}=30 \times 16 \mu m, \mathrm{n}=10)$, thick-walled, initially hyaline, aseptate with longitudinal striations on immature conidia, oval, both ends broadly rounded. 


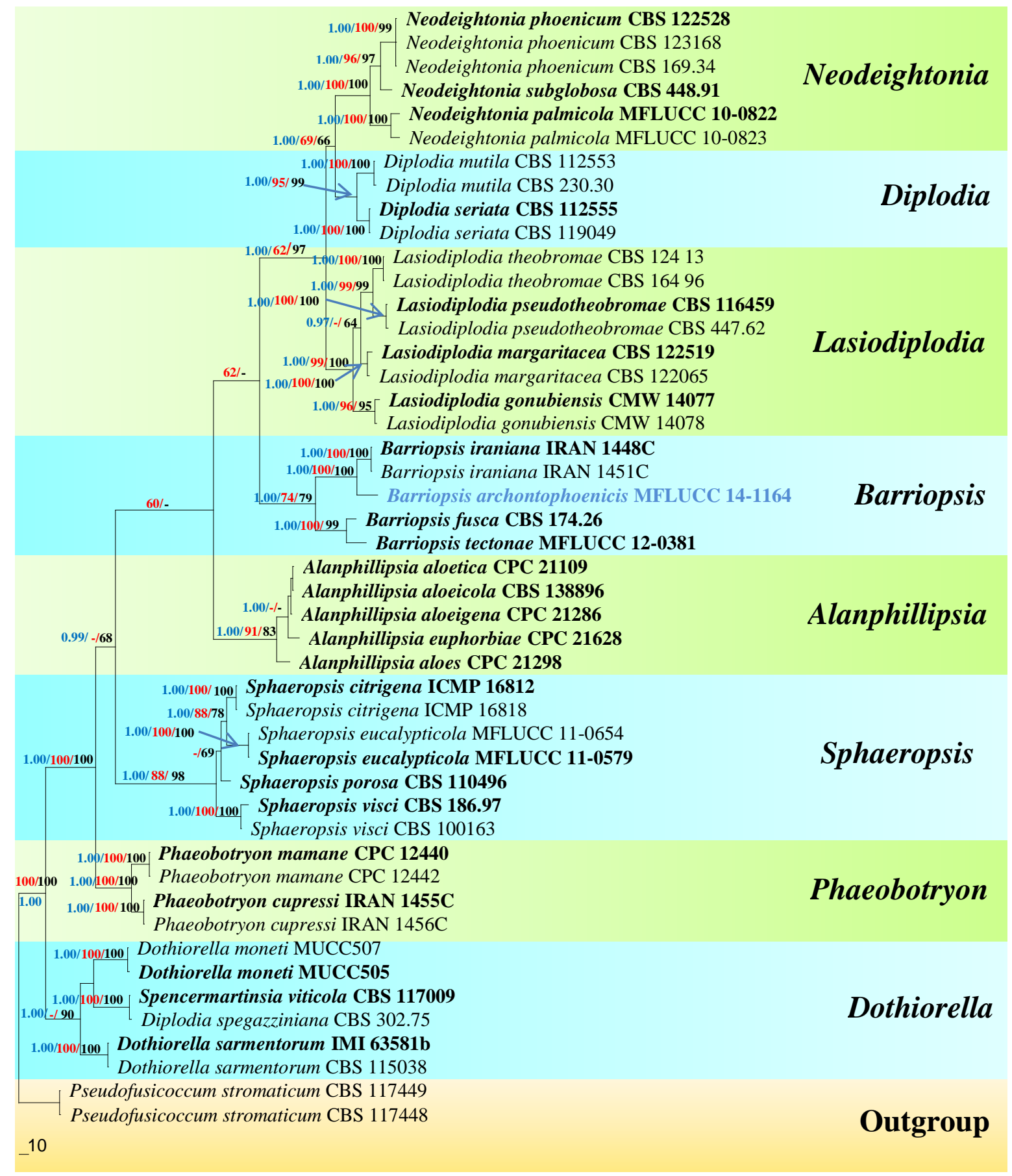

Fig. 1 - Maximum Parsimony (MP) tree derived from a combined ITS, LSU, SSU and TEF1- $\alpha$ sequence dataset. Bootstrap support values for maximum likelihood (ML, red), maximum parsimony (MP, black) greater than $60 \%$ and Bayesian posterior probabilities (BYPP, blue) greater than 0.95 are given at the nodes. The tree is rooted with Pseudofusicoccum stromaticum. The strain numbers are mentioned after the species names. The new species is indicated in blue and ex-type strains are indicated in black bold.

Culture characters - Ascospores germinating on MEA within 24 hours and germ tube produced from one end of the ascospore. Colonies on MEA and PDA fast growing, reaching 7-8.5 $\mathrm{cm}$ diam. after 4 days at $25^{\circ} \mathrm{C}$, white at the edge, grey in the middle with strong radiations outwards. After 24 days of incubation, colonies on MEA and PDA become grey-olivaceous, spongy, hyphae septate, branched, smooth, producing conidiomata and after 6 months of incubation, producing ascomata and ascospores. 

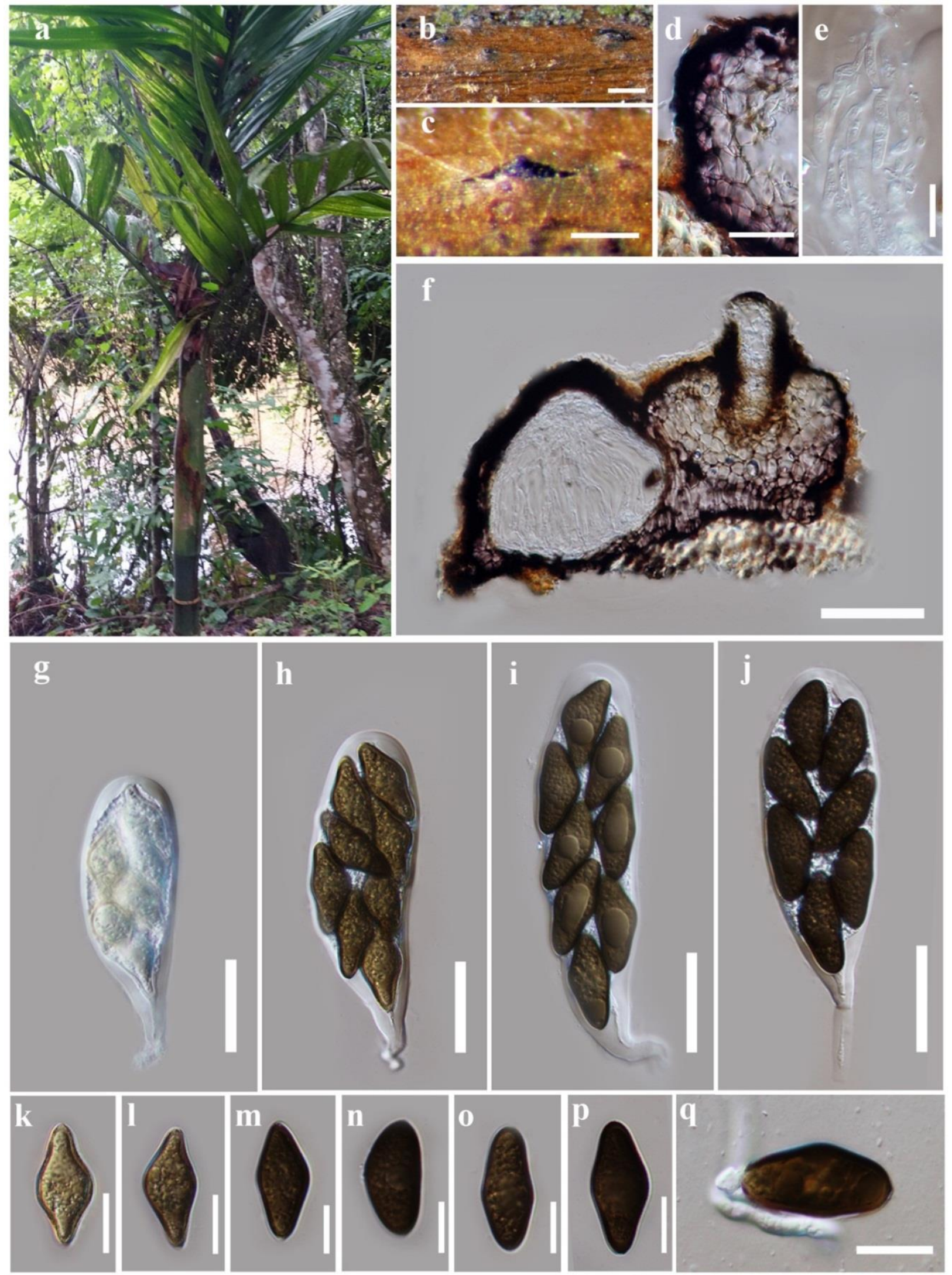

Fig. 2 - Barriopsis archontophoenicis (MFLU: 15-0015, holotype) a Palm Archontophoenix alexandrae. b Appearance of ascostromata on host substrate. c Close up of ascostroma erumpent through the host surface. d Peridium. e Pseudoparaphyses. f Section of ascostromata. g Immature ascus. $\mathrm{h}-\mathrm{j}$ Mature asci. $\mathrm{k}-\mathrm{p}$ Ascospores. $\mathrm{q}$ Germinating ascospore. Scale bars: $\mathrm{b}=500 \mu \mathrm{m}, \mathrm{c}=200$ $\mu m, \mathrm{~d}=50 \mu m, \mathrm{e}, \mathrm{g}-\mathrm{j}=20 \mu m, \mathrm{f}, \mathrm{k}-\mathrm{q}=10 \mu m$. 

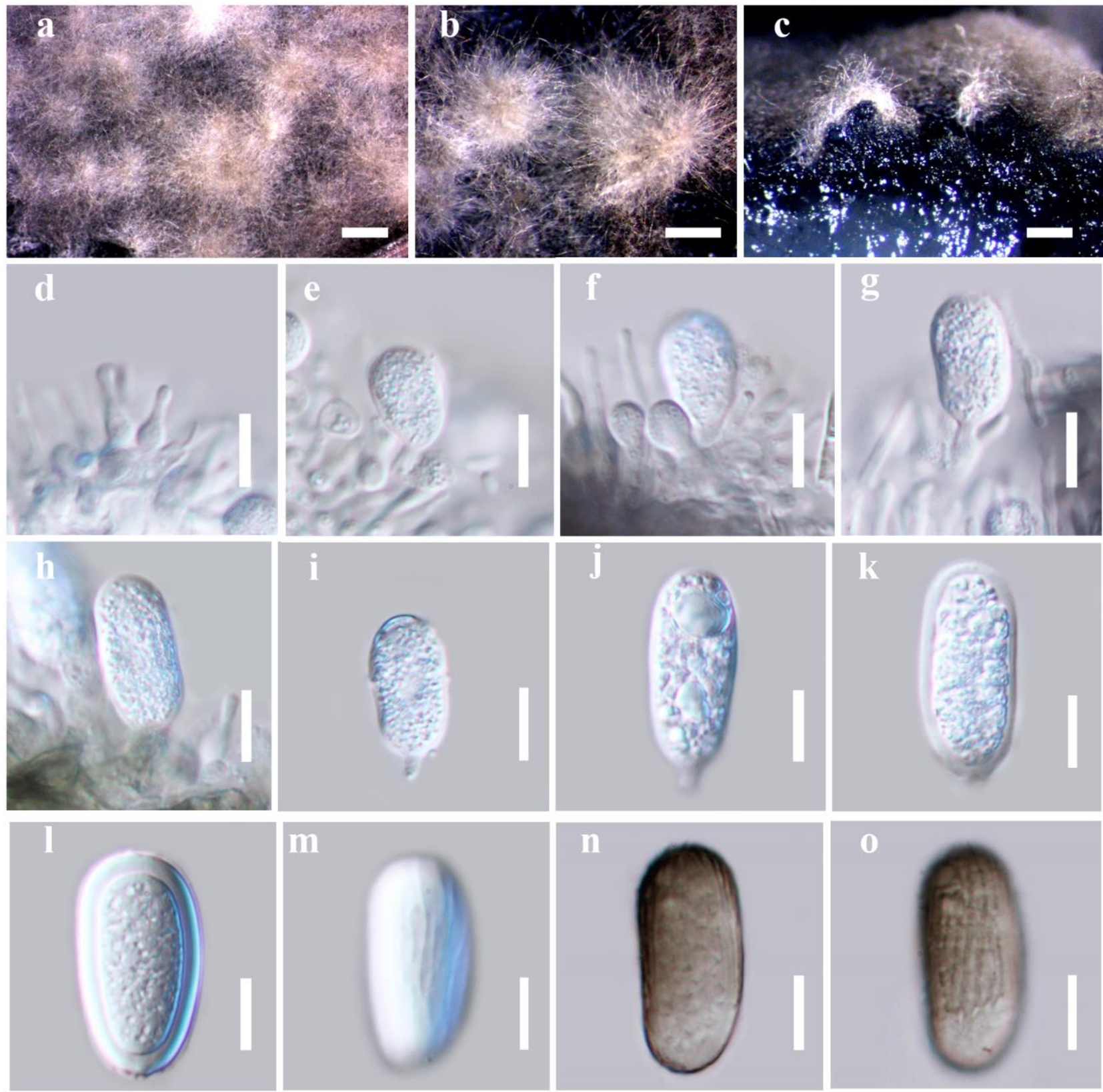

Fig. 3 - Barriopsis archontophoenicis (ex-type culture) a Conidiomata on PDA media after 26 days. b, c Close up of conidiomata. $\mathrm{d}-\mathrm{h}$ Conidia developing on conidiogenous cells. $\mathrm{f}$. Developing conidia and paraphyses. $\mathrm{i}-1$, Immature conidium. m Hyaline, immature, striate conidia. n Mature conidium. o Brown, mature, striate conidia. Scale bars: $\mathrm{a}=1,000 \mu \mathrm{m}, \mathrm{b}-\mathrm{c}=500 \mu \mathrm{m}, \mathrm{d}-\mathrm{O}=10 \mu \mathrm{m}$.

Material examined - THAILAND, Chiang Mai Province, Mushroom Research Centre, on dead woody palm (Archontophoenix alexandrae (F. Muell.) H.Wendl. \& Drude, Arecaceae), 15 August 2014, S. Konta P02a (MFLU 15-0015, holotype, HKAS92527, isotype; ex-type living culture, MFLUCC 14-1164, MUCL 55901).

Notes - Barriopsis archontophoenicis is introduced as a novel species in Barriopsis based on morphology of the sexual and asexual morphs and phylogenetic analyses. Conidia of $B$. archontophoenicis are longer than in B. fusca, shorter than B. tectonae but similar to those of $B$. iraniana. Conidia of $B$. iraniana become 1-3 septate while conidia of $B$. archontophoenicis remain aseptate even long after they are formed. Ascospores of $B$. archontophoenicis are smaller than in $B$. fusca and $B$. tectonae. No sexual morph has been reported for $B$. iraniana. Phylogenetically $B$. archontophoenicis is most closely related to $B$. iraniana, but forms a distinct lineage. 


\section{Discussion}

In this paper we introduce Barriopsis archontophoenicis as a new species. Phylogenetically it forms a distinct lineage sister to $B$. iraniana. Morphologically these two species are very similar but the aseptate conidia of $B$. archontophoenicis differentiate it from $B$. iranaiana, which has 1-3 septate conidia.

Barriopsis is a member of the family Botryosphaeriaceae (Botryosphaeriales, Liu et al. 2014) with B. fusca (N.E. Stevens) A.J.L. Phillips et al. as the type species (Phillips et al. 2008). There are presently two other accepted species, B. iraniana Abdoll et al. (Abdollahzadeh et al. 2009, Phillips et al. 2013) and B. tectonae Doilom et al. (Doilom et al. 2014) in the genus. The sexual morph of Barriopsis has brown aseptate ascospores that are widest in the middle, and lack terminal apiculi (Phillips et al. 2008). This feature was considered unique to Barriopsis differentiating it from all other genera in the Botryosphaeriaceae (Phillips et al. 2008). This feature was also seen in $B$. archontophoenicis.

Interestingly, B. archontophoenicis forms the sexual morph in culture after long periods of incubation up to 6 months. Although this has never been reported for other species in this genus it is not clear if very old cultures have ever been studied. Furthermore, within the Botryosphaeriaceae production of the sexual morph in culture is extremely rare and has been reported only for Neodeightonia globosa (Punithalingam 1969) and Neofusicoccum luteum (Denman 2002).
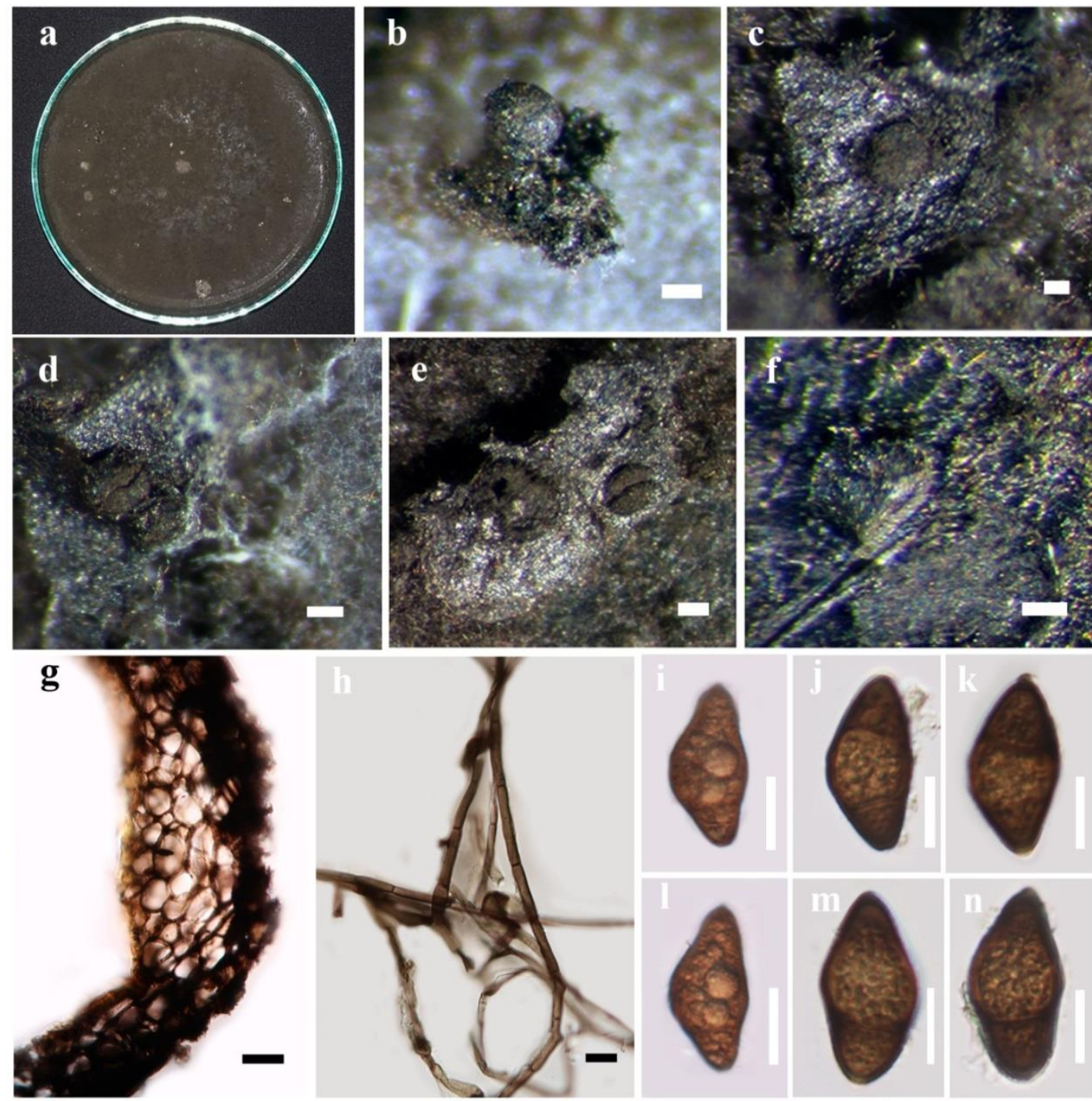

Fig. 4 - Sexual state of Barriopsis archontophoenicis (from ex-type culture) a Culture on MEA after 14 days. b-f Ascostromata formed in MEA. g Peridium. h Mycelium. i-n Ascospores. Scale bars: $\mathrm{b}-\mathrm{f}=100$ $\mu m, \mathrm{~g}=50 \mu m, \mathrm{~h}, \mathrm{i}-\mathrm{n}=10 \mu m$. 
Table 2 Ascomata, hamathecium, asci and ascospore dimensions, and host range of Barriopsis species

\begin{tabular}{|c|c|c|c|c|c|c|}
\hline Species name & Ascomata & Hamathecium & Asci & Ascospores & Host & Remarks \\
\hline $\begin{array}{l}\text { Barriopsis archontophoenicis } \\
\text { MFLU: } 15-0015 \text { (specimen) }\end{array}$ & $\begin{array}{l}\text { 99-124 } \mu m \text { diam. } \times \\
103-194 \mu m \text { high }\end{array}$ & $2-3 \mu m$ wide & $70-144 \times 17-25 \mu m$ & $17-22 \times 7-10 \mu m$ & $\begin{array}{l}\text { Archontophoenix } \\
\text { alexandrae (palm) }\end{array}$ & This study \\
\hline $\begin{array}{l}\text { Barriopsis archontophoenicis } \\
\text { MFLUCC 14-1164 (in culture) }\end{array}$ & $\begin{array}{l}206-478 \mu m \text { high } \times \\
322-435 \mu m \text { diam. }\end{array}$ & - & - & $\begin{array}{l}20-36 \times 10-16 \mu m \\
\text { 2-septate, with } \\
\text { terminal apiculi }\end{array}$ & $\begin{array}{l}\text { Archontophoenix } \\
\text { alexandrae (palm) }\end{array}$ & This study \\
\hline Barriopsis fusca (type species) & $\begin{array}{l}(430-) 546.5-520 \mu m \\
\text { diam. } \times 328-349 \mu m \\
\text { high }\end{array}$ & 3-4.5 $\mu m$ wide & $180-125 \times 30-36 \mu m$ & $\begin{array}{l}(30-) 31-36.5(-38.5) \\
\times(15.5-) 16-18.5(- \\
21) \mu m\end{array}$ & Citrus sp. & $\begin{array}{l}\text { Phillips et al. } 2008 \\
\text { Phillips et al. } 2013 \\
\text { Liu et al. } 2012\end{array}$ \\
\hline Barriopsis tectonae & $\begin{array}{l}(195-) 280-325(- \\
365) \mu m \text { high } \times(230- \\
) 265-285(-320) \mu m \\
\text { diam.- ostiole with } \\
\text { periphyses }\end{array}$ & $2.5-6 \mu m$ wide & $\begin{array}{l}(120-) 167-185(-200) \\
\times(28-) 30.5-32(-35) \\
\mu m\end{array}$ & $\begin{array}{l}(26-) 29-30(-33) \times \\
(13-) 14.5-15(-17) \\
\mu m\end{array}$ & $\begin{array}{l}\text { Tectona grandis } \\
\text { (teak) }\end{array}$ & Doilom et al. 2014 \\
\hline
\end{tabular}

Table 3 Conidiogenous cells and conidia dimensions of Barriopsis species

\begin{tabular}{|c|c|c|c|}
\hline Species name & Conidiogenous cells & Conidia & Remark \\
\hline $\begin{array}{l}\text { Barriopsis archontophoenicis } \\
\text { MFLUCC 14-1164 }\end{array}$ & $6-11 \times 3-4 \mu m$ & $26-34 \mu m \times 14-17 \mu m(\overline{\mathrm{x}}=30 \times 16 \mu m, \mathrm{n}=10)$ & This study \\
\hline Barriopsis fusca (type species) & $7-12 \times 3-5 \mu m$ & $(20-) 23-25(-28) \mu m \times(11-) 12-13(-16) \mu m$ & $\begin{array}{l}\text { Phillips et al. } 2008 \\
\text { Phillips et al. } 2013 \\
\text { Liu et al. } 2012\end{array}$ \\
\hline Barriopsis tectonae & - & $\begin{array}{l}(29-) 37-37.5(-38) \mu m \times 15.5-17.5(-18) \mu m(\overline{\mathrm{x}}=36 \\
\times 17 \mu m, \mathrm{n}=10)\end{array}$ & Doilom et al. 2014 \\
\hline Barriopsis iraniana & $7-12 \times 3-5 \mu m$ & $(22.5-) 24-30 \mu m \times(12.8-) 14-18(-21.5) \mu m$ & $\begin{array}{l}\text { Abdollahzadeh et al. } \\
2009\end{array}$ \\
\hline
\end{tabular}




\section{Acknowledgements}

This work was supported by the International Research Group Program (IRG-14-27), Deanship of Scientific Research, King Saud University, Saudi Arabia. Sirinapa Konta would like to thank the National Research Council of Thailand (grant for microfungi on palms no. 2559A30702006) for supporting studies on microfungi on palms, the Mushroom Research Foundation for supporting this research, and Kunming Institute of Botany for help in molecular work. Sirinapa Konta is grateful to Dhanushka N. Wansinghe, Wenjing Li, Saithong Kaewchaii, Ausana Mapook, Chonticha Singtripop, Mingkwan Doilom, Sinang Hongsanan, Saowaluck Tibpromma, Rungtiwa Phookamsak, Chayanard Phukhamsakda and Chada Norphunpuan for their valuable suggestions and help.

\section{References}

Abdollahzadeh J, Mohammadi GE, Javadi A, Shams-Bakhsh M, Zare R, Phillips AJL 2009 Barriopsis iraniana and Phaeobotryon cupressi: two new species of the Botryosphaeriaceae from trees in Iran. Persoonia 23, 1-8.

Ariyawansa HA, Ji-Chuan Kang, Alias SA, Chukeatirote E, Hyde KD 2014 (a) - Towards a natural classification of Dothideomycetes: The genera Dermatodothella, Dothideopsella, Grandigallia, Hysteropeltella and Gloeodiscus (Dothideomycetes incertae sedis). Phytotaxa 176, 007-017.

Ariyawansa HA, Hawksworth DL, Hyde KD, Jones EBG, Maharachchikumbura SSN, Manamgoda DS, Thambugala KM, Udayanga D, Camporesi E, Daranagama A, Jayawardena R, Liu JK, McKenzie EHC, Phookamsak R, Senanayake IC, Shivas RG, Tian Q, Xu JC. 2014 (b) Epitypification and neotypification: guidelines with appropriate and inappropriate examples. Fungal Diversity 69, 57-91.

Chomnunti P, Hongsanan S, Hudson BA, Tian Q, Persoh D, Dhami MK, Alias AS, Xu J, Liu X, Stadler M, Hyde KD 2014 - The sooty moulds. Fungal Diversity 66, 1-36.

Denman S 2002 - Botryosphaeria diseases of Proteaceae. PhD thesis, University of Stellenbosch, South Africa, p. 85.

Doilom M, Shuttleworth LA, Roux J, Chukeatirote E, Hyde KD 2014 - Barriopsis tectonae sp. nov. a new species of Botryosphaeriaceae from Tectona grandis (teak) in Thailand. Phytotaxa 176, 81-91.

Fröhlich J, Hyde KD 2000 - Palm microfungi. Fungal Diversity Research Series 3, 1-393.

Hall TA 1999 - BioEdit: a user-friendly biological sequence alignment editor and analysis program for Windows 95/98/NT. Nucleic Acids Symposium Series 41, 95-98.

Huelsenbeck JP, Ronquist F 2001 - MRBAYES: Bayesian inference of phylogenetic trees. Bioinformatics 17, 754-755.

Hyde KD 1988 - The genus Linocarpon from the mangrove palm Nypa fruticans. Transactions of the Mycological Society of Japan 29, 339-350.

Hyde KD, Taylor JE, Fröhlich 2000 - Genera of Ascomycetes from Palms. Fungal Diversity Research Series 2, 1-247.

Index Fungorum (2016) http://www.indexfungorum.org/Names/Names.asp

Jacobs K, Bergdahl DR, Wingfield MJ, Halik S, Seifert KA, Bright DE, Wingfield BD. 2004 Leptographium wingfieldii introduced into North America and found associated with exotic Tomicus piniperda and native bark beetle. Mycological Research 108: 411-418.

Jayasiri SC, Hyde KD, Ariyawansa HA, Bhat J, Buyck B, Cai L, Dai YC, Abd-Elsalam KA, Ertz D, Hidayat I, Jeewon R, Jones EBG, Bahkali AH, Karunarathna SC, Liu JK, Luangsa-ard JJ, Lumbsch HT, Maharachchikumbura SSN, McKenzie EHC, Jean-Marc Moncalvo, Ghobad-Nejhad M, Nilsson H, Ka-Lai Pang, Pereira OL, Phillips AJL, Raspé O, Rollins AW, Romero AI, Etayo J, Selçuk F, Stephenson SL, Suetrong S, Taylor JE, Tsui CKM, Vizzini A, Abdel-Wahab MA, Wen TC, Boonmee S, Dai DQ, Daranagama DA, Dissanayake AJ, Ekanayaka AH, Fryar SC, Hongsanan S, Jayawardena RS, Li WJ, Perera 
RH, Phookamsak R, de Silva NI, Thambugala KM, Tian Q, Wijayawardene NN, Zhao RL, Zhao Q, Kang JC, Promputtha I 2015 - The Faces of Fungi database: fungal names linked with morphology, phylogeny and human impacts. Fungal Diversity 74, 3-18.

Kishino H, Hasegawa M 1989 - Evaluation of the maximum likelihood estimate of the evolutionary tree topologies from DNA sequence data, and the branching order in Hominoidea. Journal of Molecular Evolution 29, 170-179.

Katoh K, Standley K 2013 - MAFFT Multiple Sequence Alignment Software Version 7: Improvements in Performance and Usability. Molecular Biology \& Evolution 30, 772-780.

Liu JK, Phookamsak R, Doilom M, Wikee S, Li YM, Ariyawansa H, Boonmee S, Chomnunti P, Dai DQ, Bhat JD, Romero AI, Zhuang WY, Monkai J, Jones EBG, Chukeatirote E, Ko Ko TW, Zhao YC, Wang Y, Hyde KD 2012 - Towards a natural classification of Botryosphaeriales. Fungal Diversity 57, 149-210.

Liu JK, Phookamsak R, Dai DQ, Tanaka K, Jones EBG, Xu JC, Chukeatirote E, Hyde KD 2014 Roussoellaceae, a new pleosporalean family to accommodate the genera Neoroussoella gen. nov, Roussoella and Roussoellopsis. Phytotaxa 181, 1-33.

Liu JK, Phookamsak R, Doilom M, Wikee S, Li YM, Ariyawansha H, Boonmee S, Chomnunti P, Dai DQ, Bhat JD, Romero AI, Zhuang WY, Monkai J, Jones EBG, Chukeatirote E, Ko Ko TW, Zhao YC, Wang Y, Hyde KD 2012 - Towards a natural classification of Botryosphaeriales. Fungal Diversity 57, 149-210.

Nylander JAA 2004 - MrModeltest v2.2. Program distributed by the author: 2. Evolutionary Biology Centre, Uppsala University: 1-2.

Page RD 1996 - TreeView: an application to display phylogenetic trees on personal computers. Computer Applications in the Biosciences 12, 357-358.

Phillips AJL, Alves A, Pennycook SR, Johnston PR, Ramaley A, Akulov A, Crous PW 2008 Resolving the phylogenetic and taxonomic status of dark-spored teleomorph genera in the Botryosphaeriaceae. Persoonia 21, 29-55.

Phillips AJL, Alves A, Abdollahzadeh J, Slippers B, Wingfield MJ, Groenewald JZ, Crous PW 2013 - The Botryosphaeriaceae: genera and species known from culture. Studies in Mycology 76, 51-167.

Phookamsak R, Norphanphoun C, Tanaka K, Dai DQ, Luo ZL, Liu JK, Su HY, Bhat DJ, Bahkali AH, Mortimer PE, Xu JC 2015 - Towards a natural classification of Astrosphaeriella-like species; introducing Astrosphaeriellaceae and Pseudoastrosphaeriellaceae fam. nov. and Astrosphaeriellopsis, gen. nov. Fungal Diversity 74, 143-97.

Phukhamsakda C, Ariyawansa HA, Phookamsak R, Chomnunti P, Bulgakov TS, Yang JB, Bhat DJ, Bahkali AH, Hyde KD 2015 - Muriphaeosphaeria galatellae gen. et sp. nov. in Phaeosphaeriaceae (Pleosporales). Phytotaxa 227, 55-65.

Punithalingam E 1969 - Studies on Sphaeropsidales in culture. Mycological Papers 119, 1-24.

Rannala B, Yang Z 1996 - Probability distribution of molecular evolutionary trees: a new method of phylogenetic inference. Journal of Molecular Evolution 43, 304-311.

Silvestro D, Michalak I 2010 - raxmlGUI: a graphical front-end for RAxML Program. And documentation available from www.sourceforge.com; Accessed August 2010.

Swofford DL 2002 - PAUP* 4.0: phylogenetic analysis using parsimony (* and other methods). Sinauer Associates, Sunderland.

Stevens NE 1926 - Two species of Physalospora on Citrus and other hosts. Mycologia 18, 206217.

Tamura K, Stecher G, Peterson D, Filipski A, Kumar S 2013 - MEGA6: molecular evolutionary genetics analysis version 6.0. Molecular Biology \& Evolution 30, 2725-2729.

Taylor WJ, Jacobson JD, Kroken S, Kasuga T, Geiser MD, Hibbett SD, Fisher CW 2000 Phylogenetic species recognition and species concepts in fungi. Fungal Genetics \& Biology 31, 21-32.

Taylor JE, Hyde KD 2003 - Microfungi of tropical and temperate palms. Fungal Diversity Research Series 12, 1-495. 
Thompson JD, Gibson TJ, Plewniak F, Jeanmougin F, Higgins DG 1997 - The CLUSTAL_X windows interface: flexible strategies for multiple sequence alignment aided by quality analysis tools. Nucleic Acids Research 25, 4876.

Vilgalys R, Hester M 1990 - Rapid genetic identification and mapping of enzymatically amplified ribosomal DNA from several Cryptococcus species. Journal of Bacteriology 172, 42384246.

White TJ, Bruns T, Lee S, Taylor J 1990 - Amplification and direct sequencing of fungal ribosomal RNA genes for phylogenetics. In: Innis, M.A, Gelfand, D.H, Sninsky, J.J. \& White, T.J. (eds) PCR protocols: a guide to methods and applications. San Diego,

Zhaxybayeva O, Gogarten JP 2002 - Bootstrap, Bayesian probability and maximum likelihood mapping: exploring new tools for comparative genome analyses. BMC Genomics 3, 4 . 